\title{
A study of gestational diabetes mellitus and fetal outcome in a tertiary care center
}

\author{
Aashka M. Mashkaria*, Babulal S. Patel, Aastha M. Mashkaria, Akshay C. Shah, \\ Shashwat K. Jani, Adwait B. Patel, Raj M. Mehta
}

Department of Obstetrics and Gynecology, Smt. N. H. L. Municipal Medical College, SVPIMSR, Ahmedabad, Gujarat, India

Received: 11 June 2020

Accepted: 08 July 2020

\section{*Correspondence:}

Dr. Aashka M. Mashkaria,

E-mail: aashkamashkaria95@gmail.com

Copyright: () the author(s), publisher and licensee Medip Academy. This is an open-access article distributed under the terms of the Creative Commons Attribution Non-Commercial License, which permits unrestricted non-commercial use, distribution, and reproduction in any medium, provided the original work is properly cited.

\section{ABSTRACT}

Background: Modern desk-bound lifestyle and unhealthy dietary changes have brought a rise in the prevalence of obesity and gestational diabetes mellitus (GDM). It is associated with severe hazards to the mother and the baby. It is mandatory that early diagnosis ensues and timely and congruous management is undertaken.

Methods: In this observational study, 1250 women were included. A standardized questionnaire was formed and their details were noted. Tests for glucose levels, complete blood picture, urine examination were performed. An oral glucose tolerance test was performed on all the patients. Neonatal outcomes in terms of birth weight and the presence of complications were noted.

Results: A total $201(16.1 \%)$ of all women were having gestational diabetes mellitus (GDM). Most mothers were in the 25-30 age group. The majority of the women had a BMI between 26-30. 21.9\% of babies were having weight $>3.5$ kgs. $11.4 \%$ of babies were $<2.5 \mathrm{kgs}$. Out of 201 neonates, 90 babies were having complications. Major complications in neonates were macrosomia and respiratory distress. Therefore, early diagnosis, glycemic control, and timely and congruous management are advantageous to both mother and baby.

Conclusions: GDM complicating the pregnancy results in a higher prevalence of complications in the mother and the neonate. Therefore, appropriate control of the sugar level in mothers is necessary and it decreases the morbidity and mortality rates in the babies as well as the mothers.

Keywords: Big baby, Fetal outcomes, Gestational diabetes mellitus, Oral glucose tolerance test

\section{INTRODUCTION}

Gestational diabetes mellitus (GDM) is defined as any degree of glucose intolerance with onset or first recognition during pregnancy. This definition applies irrespective of the form of treatment or whether diabetes persists after the pregnancy. Maternal metabolism changes substantially during pregnancy. Changes in carbohydrate and lipid metabolism occur during pregnancy to ensure a continuous supply of nutrients to the growing life despite intermittent maternal food intake. These metabolic changes are progressive and may be accentuated. The advancing pregnancy causes insulin resistance and diabetogenic stress due to placental hormones, ultimately resulting in an increase in insulin secretion. An inadequate compensation of insulin, if inadequate, may result in the maternal high blood glucose level. ${ }^{1}$ Due to modern desk-bound lifestyle and unhealthy dietary changes, there is a rise in the prevalence of obesity, so is the incidence of GDM. The prevalence of GDM varies in different regions. Overall, the incidence of GDM is estimated to be $7.1 \%$, with an annual increase of 1,80,000 cases. In the USA alone, the GDM is reported to be $12-15 \%$ of all pregnancies. ${ }^{2}$ In Europe, America, 
and Asia, in general, the prevalence is reported to be 3$7 \% .^{3-5}$

In comparison to the white women, the Indian women are prone to have GDM by eleven-fold, eightfold in South East Asia, and three-fold in Arab and Afro-Caribbean countries. $^{6}$ The complications occur in $2-3 \%$ of all pregnancies. It is also associated with a higher incidence of diabetes to the mother later on in life. ${ }^{7}$ Apart from Macrosomia, the new-born also suffers from respiratory distress, hypocalcaemia, polycythaemia, hypoglycaemia, growth restriction, hypomagnesemia, and congenital malformations. There is $42.9 \%$ mortality in infants with mothers with poor glycaemic control. ${ }^{8}$

Therefore, it is mandatory that early diagnosis ensues and timely and congruous management is undertaken.

\section{METHODS}

This observational study was conducted in the department of obstetrics and gynecology at Sardar Vallabhbhai Patel Institute of Medicine Sciences and Research, Ahmedabad, Gujarat over a period of 13 months from January-2019 to April -2020. 5014 patients were screened for gestational diabetes mellitus (GDM) in our hospital.

\section{Inclusion criteria}

- All the pregnant women who were included in the study were in their 24-28 weeks of gestation and they were followed up till their termination.

\section{Exclusion criteria}

- Patients with a history of diabetes prior to the present study

- Gestational diabetes in a previous pregnancy

- History of stillbirth or fetal death

- Pregnancy with more than one fetus

- Known major fetal anomaly

- Current or planned corticosteroid therapy

- Asthma requiring medication

- Current or planned beta-adrenergic therapy.

The study procedure was explained clearly to the patients and informed consent was taken from all of them.

A standardized questionnaire was formed and details regarding the age, weight, body mass index (BMI), parity, previous medical and obstetrics history and familial history of diabetes details like age, weight, body mass index (BMI), parity, medical history, previous history and familial history of diabetes, obstetric history were noted. This was followed by complete physical examination, tests for glucose levels, complete blood picture, routine urine examination.
Oral glucose tolerance test (OGTT) was done after an overnight fast. $75 \mathrm{~g}$ of pure glucose was mixed with 100 $\mathrm{ml}$ of water and the patient was asked to drink this mixture. $2 \mathrm{ml}$ of blood was collected in a sodium fluoride oxalate bottle immediately. This was considered as 0hour time. Blood samples were collected again at 1, 2, and 3 hours respectively. Plasma glucose levels were interpreted using the NDDG (National Diabetes Data Group) criteria.

\section{RESULTS}

Figure 1, a total of 1250 pregnant women attending the outpatient and the inpatient wards of the study hospital were included in the study as per our inclusion criteria.

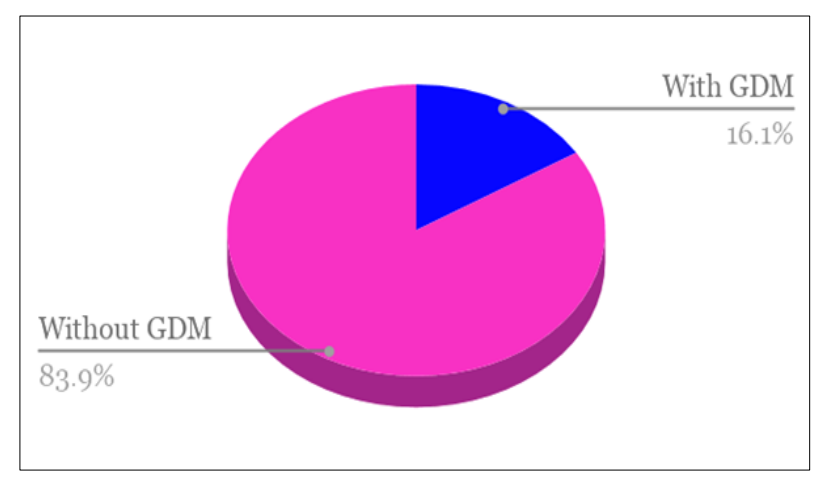

Figure 1: Prevalence of GDM.

Table 1: Demographic details.

\begin{tabular}{|ll|l|}
\hline Details & Number $(\mathbf{N}=201)$ & $\%$ \\
\hline Age (years) & & \\
\hline$<25$ & 23 & $11.4 \%$ \\
\hline $25-30$ & 141 & $70.1 \%$ \\
\hline$>30$ & 37 & $18.5 \%$ \\
\hline Gravida & \\
\hline Primi & 82 & $40.8 \%$ \\
\hline Multigravida & 119 & $59.2 \%$ \\
\hline BMI & & \\
\hline$<18$ & 30 & $14.9 \%$ \\
\hline $18-24$ & 45 & $22.4 \%$ \\
\hline $24-30$ & 86 & $42.8 \%$ \\
\hline$>30$ & 40 & $19.9 \%$ \\
\hline
\end{tabular}

Out of all, 215 patients had abnormal fasting and postprandial blood sugar levels and were tested for OGTT. All the $201(16.1 \%)$ of 1250 were positive for OGTT and were considered to have gestational diabetes mellitus (GDM).

Table 1, most of the patients were in the 25-30 age group $(70.1 \%)$. Around $11.4 \%$ of them were below the age of 25 years. $82(40.8 \%)$ were pregnant for the first time and $119(59.2 \%)$ were multi gravid. 45 patients $(22.4 \%)$ had a BMI of $18-24$, while $40(19.9 \%)$ has an index of $>30$. 
However, the majority of the patients had a BMI between 26-30 (Table 1).

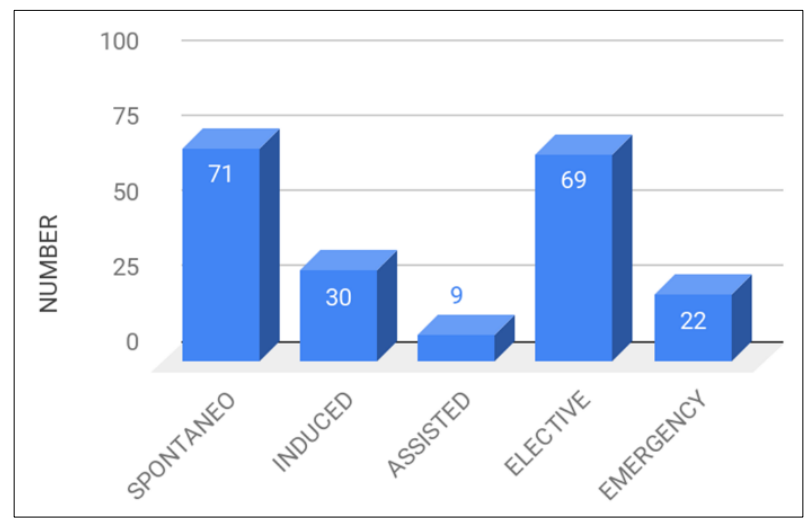

Figure 2: Mode of delivery.

Figure 2, the most predominant mode of delivery of the women was spontaneous (35.3\%). 69 patients (34\%) had opted for elective surgery. The emergency procedure was performed in 22 patients $(10.9 \%$ ) (Figure 2$)$.

Figure 3, there were no neonatal deaths in this study. Most of the babies had a birth weight of above $3 \mathrm{kgs}$. Out of them, $100(49.8 \%)$ had a birth weight between 3-3.5 kgs. $<2.5 \mathrm{kgs}$ were seen in $23(11.4 \%)$ patients. $21.9 \%$ of babies were considered as big babies (Figure 3).

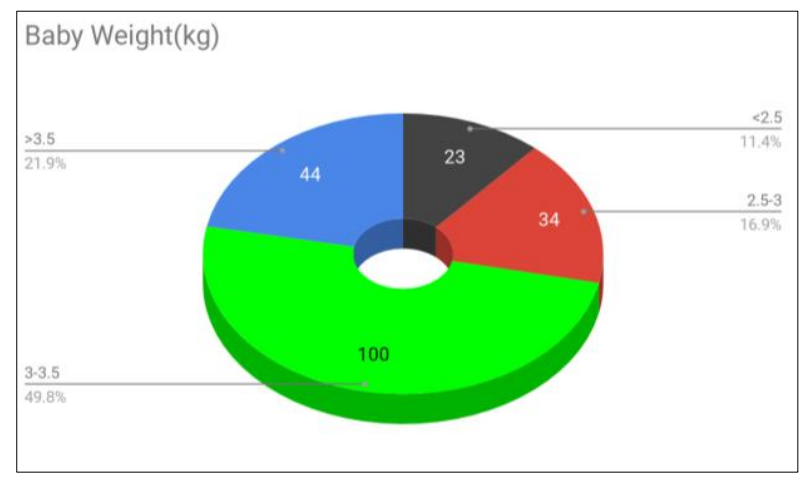

Figure 3: Birth weight of the neonate.

Table 2: Prevalence of complications in neonates.

\begin{tabular}{|ll|}
\hline Complication \\
\hline Babies without any complications & 111 \\
\hline Babies with minor/major complications & 90 \\
\hline
\end{tabular}

Table 2, out of 201 babies, $55.2 \%$ of babies were having no complications. $44.8 \%$ of babies were having minor or major complications like - macrosomia, hypocalcaemia, hypoglycaemia, respiratory distress, congenital anomalies, neonatal jaundice.

Figure 4, out of 201 neonates, 111 babies were having no complications, and Apgar score at 1 and 5 minutes were within the normal range according to the neonatologist.

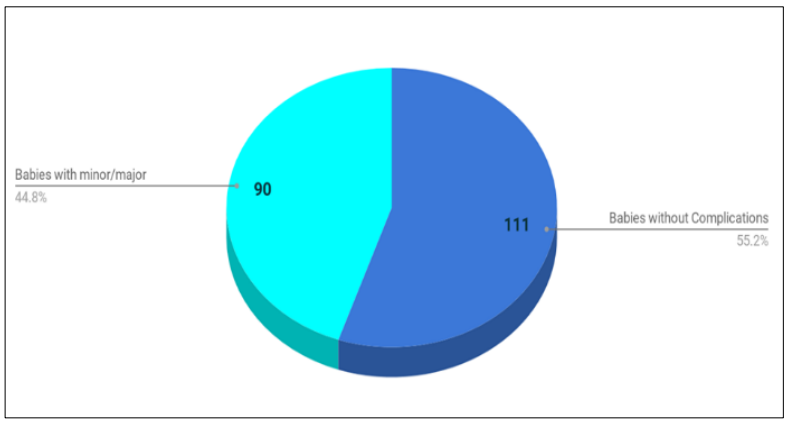

Figure 4: Prevalence of complications in neonates.

There were no neonatal deaths or stillbirths in our studies. 90 babies were having complications like- macrosomia, hypocalcaemia, hypoglycaemia, Respiratory distress, congenital anomalies, neonatal jaundice.

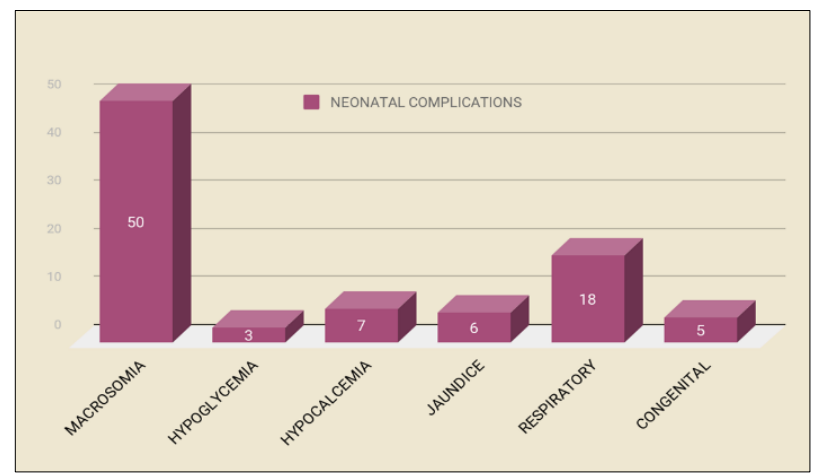

Figure 5: Neonatal complications.

Figure 5, macrosomia was the most common complication among all the 201 neonates (24.8\%). Respiratory distress was observed in 18 neonates $(8.95 \%)$. Other complications that were observed were hypoglycaemia, hypocalcaemia, and neonatal jaundice. Babies born with congenital anomaly were seen in 5 (2.48\%) (Figure 5).

\section{DISCUSSION}

Gestational diabetes mellitus (GDM) is defined as any degree of glucose intolerance with onset or first recognition during pregnancy. It appears usually during pregnancy, but disappears soon after, probably because of the hormonal and metabolic changes during pregnancy. Normally, the impaired insulin resistance and/or reduced glucose utilization due to a cellular defect leads to reduced insulin secretory capacity of the woman. This may lead to at a later stage, fully-fledged diabetes in the woman. Often, it is a result of risk factors such as for overweight, sedentary lifestyle, advanced age, and previous history of GDM. ${ }^{9-11}$

The prevalence of GDM is estimated to be between 14$17 \%$ in India. The present study has shown a prevalence of $16.1 \%$ of GDM in women in our area. A prevalence of $8.6 \%$ in mothers was noted in a study by Malak, in the 
Kingdom of Saudi Arabia. ${ }^{12} 4.2 \%$ incidence was reported in a study by Dudhwadkar and Fonseca from Mumbai. ${ }^{13}$ In other similar studies, such as Nilofer in Karnataka, a rate of $6 \%$ and Wahi et al from Jammu came up with a rate of $6.94 \% .^{14,15}$

The most common age group affected in this study was 25-30 years, which was much greater than patients below 25 years of age. A similar result was observed by Rajput et al, who reported an increase of 3.8 times more in women of greater than 25 years rather than lesser. ${ }^{16} \mathrm{~A}$ similar observation was made by Seshaiah et al in their study. ${ }^{17}$

The BMI of most of the patients in this study was between 24-30. A strong association was noticed in the present study between obesity and GDM. This was in accordance with many other studies. ${ }^{17-20}$ Primigravida was seen in $40.8 \%$ of the patients, while it was observed that on the increase in the parity, the risk of GDM increases. Similar reports were observed by Dudhwadkar et al and Rajput et al. ${ }^{13,16}$

A total $35.3 \%$ of the mothers in this study had a normal spontaneous vaginal delivery, while $34 \%$ had elective cesarean sections. Most of the patients who went in for the caesarian section had babies with macrosomia. The incidence of macrosomia was the prime complication among the neonates of mothers having GDM. A similar result was reported by Odar et al, wherein the number of vaginal deliveries and elective surgeries was similar though the number of cesarean sections was indicated more in the case of macrosomia babies. ${ }^{21}$ However, in a few studies, there was an increase in the caesarean sections compared to normal deliveries. . $2,23^{23}$

\section{CONCLUSION}

Multigravida women, elderly women should be extra cautious in their present pregnancy. Women having past history of GDM should be extra careful in consequent pregnancies. A simple blood test is useful for the early detection of GDM and early congruous management of the disease. Appropriate control of sugar level in the mother can decrease the morbidity and mortality rates of the baby as well as the mother, thereby lowering the hospital burden and individual cost. Women with obesity in their first pregnancy also need to take applicable preventive measures.

\section{Funding: No funding sources}

Conflict of interest: None declared

Ethical approval: The study was approved by the Institutional Ethics Committee

\section{REFERENCES}

1. Seshiah V, Das AK, Balaji V, Joshi SR, Parikh MN, Gupta S. Diabetes in the pregnancy study group. gestational diabetes mellitus-guidelines. J Assoc Physicians India. 2006;54:622-8.

2. Kim C, Newton KM, Knopp RH. Gestational diabetes and the incidence of type 2 diabetes: a systematic review. Diabetes Care. 2002;25:1862-8.

3. Akhtar J, Qureshi R, Rahim F, Moosvi S, Rehman A, Jabbar A, et al. Diabetes in pregnancy in Pakistani women: prevalence and complications in an indigenous South Asian community. Diab Med. 1996;13(3):189-91.

4. Bancrofti K, Tuffnel GC, Mason GC, Rogerson LJ, Mansfield M. A randomized controlled pilot study for the management of gestational impaired glucose tolerance. BJOG. 2000;107:959-63.

5. Young C, Kuehl TJ, Sulak PJ, Allen RS. GDM screening in subsequent pregnancy of previously healthy patients. Am J Obstet Gynecol. 2000;181(4):798-802.

6. Dornhost A, Pateson EM, Nicholls JSD, Wadsworth J, Chiu DC, Elkeles RS, et al. High prevalence of GDM in women from ethnic minority groups. Diab Med. 1992;9:820-5.

7. Davey RX, Hamblin PS. Selective versus universal screening for gestational diabetes mellitus: An evaluation of predictive risk factors. Med J Aust. 2001;174:118-21.

8. Otolorin EO, Famuyiwa OO, Bella AF, Dawodu AH, Adelusi B. Reproductive performance following active management of diabetic pregnancies at the university college hospital, Ibadan, Nigeria. Afr J Med Med Sci. 1985;14:155-60.

9. King H. Epidemiology of glucose intolerance and gestational diabetes in women of child bearing age. Diabetes Care. 1998;21(Suppl 2):9-13.

10. Engelgau MM, Herman WH, Smith PJ, German RR, Aubert RE. The epidemiology of diabetes and pregnancy in the US, 1988. Diab Care. 1995;18(7):1029-33.

11. Shefali AK. Pregnancy outcomes in pre-gestational and gestational diabetic women in comparison to a non-diabetic women-a prospective study in Asian Indian mothers. $\mathrm{J}$ Assoc Physicians India. 2006;54:613-8.

12. Al-Hakeem MM. Pregnancy outcome of gestational diabetic mothers: experience in a tertiary center. J Fam Community Med. 2006;13(2):55-9.

13. Dudhwadkar AR, Fonseca MN. Maternal and fetal outcome in gestational diabetes mellitus. Int $\mathbf{J}$ Reprod Contracept Obstet Gynecol. 2016;5:3317-21.

14. Nilofer AR, Raju VS, Dakshayini BR, Zaki SA. Screening in a high-risk group of gestational diabetes mellitus with its maternal and fetal outcomes. Indian J Endocrinol Metab. 2012;16:74-8.

15. Wahi P, Dogra V, Jandial K, Bhagat R, Gupta R, Gupta S, et al. Prevalence of gestational diabetes mellitus and its outcomes in the Jammu region. J Assoc Physicians India. 2011;59:227-30.

16. Rajput R, Yadav Y, Nanda S, Rajput M. Prevalence of gestational diabetes mellitus and associated risk 
factors at a tertiary care hospital in Haryana. The Indian J Med Res. 2013;137(4):728.

17. Seshiah V, Balaji V, Balaji MS, Paneerselvam A, Arthi T, Thamizharasi M, et al. Prevalence of gestational diabetes mellitus in South India (Tamil Nadu): a community-based study. J Assoc Physicians India. 2008;56:329-33.

18. Soheilykhah S, Mogibian M, Rahimi-Saghand S, Rashidi M, Soheilykhah S, Piroz M. Incidence of gestational diabetes mellitus in pregnant women. Inter J Reprod BioMed. 2010;8(1):24-8.

19. Sreekanthan K, Belicita A, Rajendran K, Vijayakumar A. Prevalence of Gestational diabetes mellitus in a medical college in South India: a pilot study. Indian J Clin Pract, 2014;25(4):342-7.

20. Swami SR, Mehetre R, Shivane V, Bandgar TR, Menon PS, Shah NS. Prevalence of carbohydrate intolerance of varying degrees in pregnant females in western India (Maharashtra): A hospital-based study. J Indian Med Assoc. 2008;106:712-4.

21. Odar E, Wandabwa J, Kiondo P. Maternal and fetal outcome of gestational diabetes mellitus in Mulago Hospital, Uganda. Afr Health Sci. 2004;4(1):9-14.

22. Naylor CD, Sermer M, Chen E, Sykora K. Caesarian delivery in relation to birth weight and glucose gestational tolerance. JAMA. 1996;275(15):116470 .

23. Coustan D. Management of GDM: a self-fulfilling prophecy? JAMA. 1996;275:895-900.

Cite this article as: Mashkaria AM, Patel BS, Mashkaria AM, Shah AC, Jani SK, et al. A study of gestational diabetes mellitus and fetal outcome in a tertiary care center. Int J Reprod Contracept Obstet Gynecol 2020;9:3417-21. 